\title{
Quantum-enhanced micromechanical displacement sensitivity
}

Hoff, Ulrich Busk; Harris, Glen I.; Madsen, Lars Skovgaard; Kerdoncuff, Hugo; Lassen, Mikael Østergaard; Nielsen, Bo Melholt; Bowen, Warwick P.; Andersen, Ulrik Lund

\section{Published in:}

Optics Letters

Link to article, DOI:

10.1364/OL.38.001413

Publication date:

2013

Document Version

Publisher's PDF, also known as Version of record

Link back to DTU Orbit

Citation (APA):

Hoff, U. B., Harris, G. I., Madsen, L. S., Kerdoncuff, H., Lassen, M. Ø., Nielsen, B. M., Bowen, W. P., \& Andersen, U. L. (2013). Quantum-enhanced micromechanical displacement sensitivity. Optics Letters, 38(9), 1413-1415. https://doi.org/10.1364/OL.38.001413

\section{General rights}

Copyright and moral rights for the publications made accessible in the public portal are retained by the authors and/or other copyright owners and it is a condition of accessing publications that users recognise and abide by the legal requirements associated with these rights.

- Users may download and print one copy of any publication from the public portal for the purpose of private study or research.

- You may not further distribute the material or use it for any profit-making activity or commercial gain

- You may freely distribute the URL identifying the publication in the public portal 


\title{
Quantum-enhanced micromechanical displacement sensitivity
}

\author{
Ulrich B. Hoff, ${ }^{1, *}$ Glen I. Harris, ${ }^{2}$ Lars S. Madsen, ${ }^{1}$ Hugo Kerdoncuff, ${ }^{1}$ Mikael Lassen, ${ }^{1}$ \\ Bo M. Nielsen, ${ }^{1}$ Warwick P. Bowen, ${ }^{2}$ and Ulrik L. Andersen ${ }^{1}$ \\ ${ }^{1}$ Department of Physics, Technical University of Denmark, Fysikvej bld. 309, Lyngby 2800 Kgs., Denmark \\ ${ }^{2}$ Centre of Excellence in Engineered Quantum Systems, University of Queensland, St Lucia, Queensland 4072, Australia \\ ${ }^{*}$ Corresponding author: ulrich.hoff@fysik.dtu.dk
}

Received January 29, 2013; revised March 13, 2013; accepted March 22, 2013;

posted March 25, 2013 (Doc. ID 184455); published April 24, 2013

\begin{abstract}
We report on a hitherto unexplored application of squeezed light: for quantum-enhancement of mechanical transduction sensitivity in microcavity optomechanics. Using a toroidal silica microcavity, we experimentally demonstrate measurement of the transduced phase modulation signal in the frequency range $4-5.8 \mathrm{MHz}$ with a sensitivity $-0.72( \pm 0.01) \mathrm{dB}$ below the shot noise level. This is achieved for resonant probing in the highly undercoupled regime, by preparing the probe in a weak coherent state with phase squeezed vacuum states at sideband frequencies. (c) 2013 Optical Society of America

OCIS codes: (120.4880) Optomechanics; (140.3945) Microcavities; (270.6570) Squeezed states; (120.2920) Homodyning.
\end{abstract}

http://dx.doi.org/10.1364/OL.38.001413

Originally spurred by the field of gravitational wave detection, considerable attention has been attracted to achieving high-sensitivity interferometric detection of mechanical displacements. Within recent years, such displacement sensing techniques have also found application for cantilever-based single spin detection [1], cavity optomechanical magnetometry [2], and the quest to reveal quantum effects in mesoscopic mechanical systems [3, $\underline{3}]$. Fundamentally, the total measurement uncertainty of any optical displacement readout has contributions from two distinct noise sources [5]: imprecision noise in the form of photon shot noise of the detected probe light and quantum back-action noise due to a stochastic radiation pressure force imparted on the mechanical resonator by vacuum fluctuations entering the setup. Using coherent states of light, imprecision noise sets a limiting sensitivity $\propto 1 / \sqrt{N}$, with $N$ being the deployed number of photons, suggesting that arbitrarily high sensitivity can be achieved simply by increasing the optical power. However, as $N$ increases quantum back-action noise $\propto \sqrt{N}$ contributes more and more, and an optimal sensitivity, the so-called standard quantum limit (SQL), is reached when the two noise sources contribute equally to the total measurement imprecision at the mechanical resonance frequency. Being a context-dependent limit, the SQL is not absolute and can in principle be surpassed. In microtoroidal resonators, however, the high optical quality factor will cause the onset of dynamical back-action well before reaching the SQL. This effect was first observed in such systems by Rokhsari et al. [6] and it has recently been shown that the resulting parametric instability can be suppressed using feedback techniques [7]].

It has been long known that the injection of squeezed light can be used to improve the phase sensitivity of an interferometric measurement [8] and this has been experimentally verified for both Mach-Zehnder [9], Sagnac [10], and large-scale gravitational-wave [11] interferometer topologies. Also, squeezed-light-enhanced microparticle tracking has recently been demonstrated [12].
In this Letter, we report on experimental demonstration of squeezed-light-enhanced mechanical transduction sensitivity using a tapered fiber coupled microtoroidal resonator at room temperature. With this implementation of quantum-enhanced interferometry, we have extended the applicability of the technique to the regime of micromechanical oscillators. The potential benefits of using squeezed light are numerous, e.g.: (i) it improves the minimum achievable sensitivity at a given probe power, thus providing a way to effectively shift the onset of dynamical back-action that would otherwise mask the improved imprecision noise as the power is ramped up; (ii) it shifts the SQL to experimentally more feasible power levels; and (iii) quadrature anticorrelations can in principle be exploited to beat the SQL by using an optimized squeezing phase [13].

Cavity optomechanics with microtoroidal resonators [14] have been proven to be a promising system for high-sensitivity displacement detection [15]. Such systems integrate a high- $Q$ optical mode with a mechanical harmonic oscillator and couple the two via the radiation pressure mediated optomechanical interaction, formally described by the Hamiltonian $H_{\mathrm{om}}=\hbar g x a^{\dagger} a$. Here, $x$ is the mechanical position operator, $a$ and $a^{\dagger}$ are the optical ladder operators, and $g=\partial \omega_{0} / \partial x$ is the optomechanical coupling coefficient describing the linear change in optical resonance frequency for small displacements $\delta x$ from the mechanical equilibrium position. For a thermally driven mechanical degree of freedom $H_{\mathrm{om}}$ leads to harmonic modulation of the optical resonance frequency, with modulation index given by the peak frequency deviation to modulation frequency ratio $\xi=g \delta x / \Omega_{m}[14]$. For resonant optical excitation, this translates into a pure phase modulation of the transmitted light field at the mechanical oscillation frequency $\Omega_{m}$. In the sideband picture (Fig. 1), this is formally equivalent to displacements $D_{\omega_{0} \pm \Omega_{m}}[i \xi \alpha / \sqrt{2}]$ of the $\omega_{0} \pm \Omega_{m}$ sideband modes, where $\alpha$ is the coherent carrier amplitude. Thus, the optomechanical interaction results in the excitation of weak coherent sideband states carrying information 


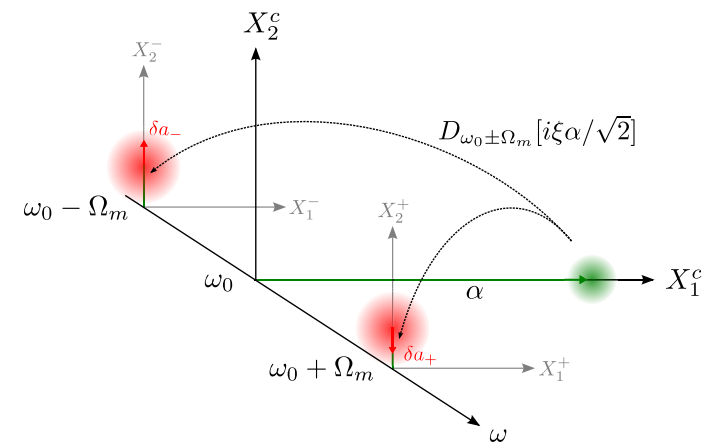

Fig. 1. Combined sideband and phase space representation of the optomechanical transduction mechanism. The optomechanical phase modulation transfers photons from the coherent carrier (green) into the sidebands, displacing the quantum states $\delta a_{ \pm}$(red).

about the mechanical oscillation frequency and displacement amplitude. This encoded information can be extracted by subsequent homodyne detection of the phase quadrature. The detection process is a joint measurement of the upper and lower sideband states at each detection frequency $\Omega$, and the resulting measurement imprecision noise, given by the spectral variance of the recorded phase quadrature, is [16]

$$
V\left(X_{2}[\Omega]\right) \propto \beta^{2}\left(V\left(X_{2}^{+}+X_{2}^{-}\right)+V\left(X_{1}^{+}-X_{1}^{-}\right)\right),
$$

where $\beta$ is the local oscillator (LO) amplitude. If the sideband states are initially vacua, the transduced signal will be measured with one shot noise unit of noise. Alternatively, quantum correlated sidebands can be added symmetrically around the carrier, forming phase squeezed vacuum states. In this case the measurement imprecision noise is reduced below the shot noise level (SNL), yielding a quantum-enhanced displacement sensitivity.

In the experiment, as shown schematically in Fig. 2, light at $\lambda=1064 \mathrm{~nm}$ from an Nd:YAG laser is first spectrally and spatially filtered by a mode cleaning cavity and subsequently split on a polarizing beam splitter to form probe and LO fields. The probe field can follow two alternative beam paths: path 1 for coherent state probing and path 2 for generation of phase squeezed vacuum sideband states, in a PPKTP-based optical parametric amplifier (OPA) [17]. The probe field is coupled into a bare optical fiber using a single antireflection-coated aspheric singlet lens with $f=8.07 \mathrm{~mm}$. For evanescent coupling to the microtoroidal resonator, the bare fiber is tapered down to a diameter of approximately $1 \mu \mathrm{m}$ over a region of $20 \mathrm{~mm}$, using a hydrogen-flame brushing technique. The transmitted probe field is then interfered with the LO and guided into a balanced homodyne detector. The differential homodyne signal is spectrally analyzed using an electronic spectrum analyzer (ESA). The individual dc components from the homodyne detectors are fed to a proportional-integral (PI) controller, actively stabilizing the LO phase via a piezo-actuated mirror.

To characterize the squeezed state transmitted through the tapered fiber, we decouple the toroid and perform homodyne measurements of the field quadratures by linearly sweeping the LO phase. The recorded data are presented in the inset of Fig. 3. Linear fits to vacuum

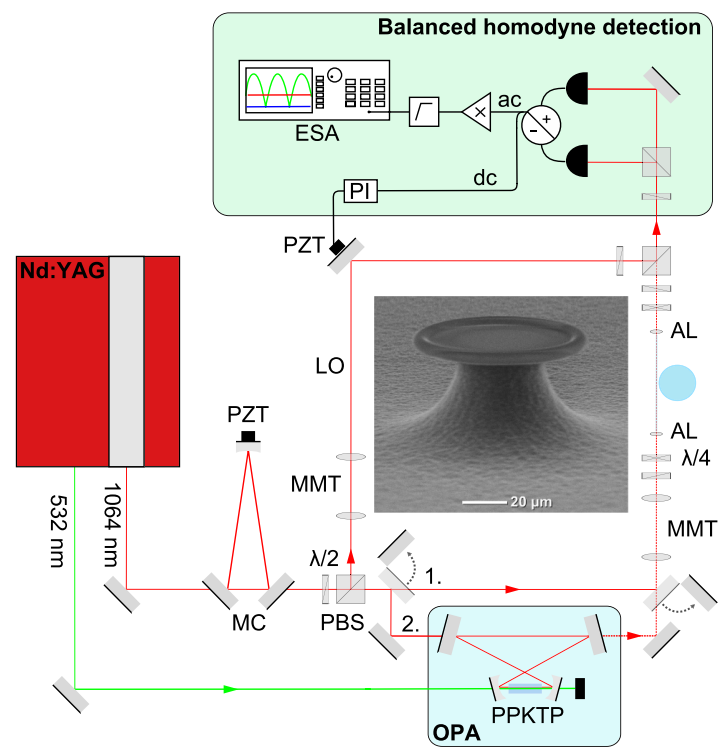

Fig. 2. Schematic diagram of the experimental setup. MC, mode cleaning cavity; PZT, piezoelectric transducer; PBS, polarizing beam splitter; MMT, mode matching telescope; LO, local oscillator field for balanced homodyne detection; AL, aspherical lens. Inset: SEM micrograph of the toroidal microcavity with major diameter $\simeq 60 \mu \mathrm{m}$ and minor diameter $\simeq 6 \mu \mathrm{m}$. At $1064 \mathrm{~nm}$ the free spectral range is $1.04 \mathrm{THz}$.

and squeezed noise levels yields $-1.20( \pm 0.03) \mathrm{dB}$ of squeezing, corresponding to a squeezing factor $r=$ $0.138( \pm 0.003)$, limited by large coupling and propagation losses in the tapered fiber amounting to $\gtrsim 30 \%$. We attribute the majority of the losses to adhesion of dust [18] and water to the fiber, causing increased scattering from the tapered region. The homodyne visibility was $98( \pm 1) \%$ and the quantum efficiency of photodiodes (Epitaxx ETX-500) is $87( \pm 2) \%$.

We establish optical coupling to the toroid by bringing it in the proximity of the tapered fiber. Coarse thermal

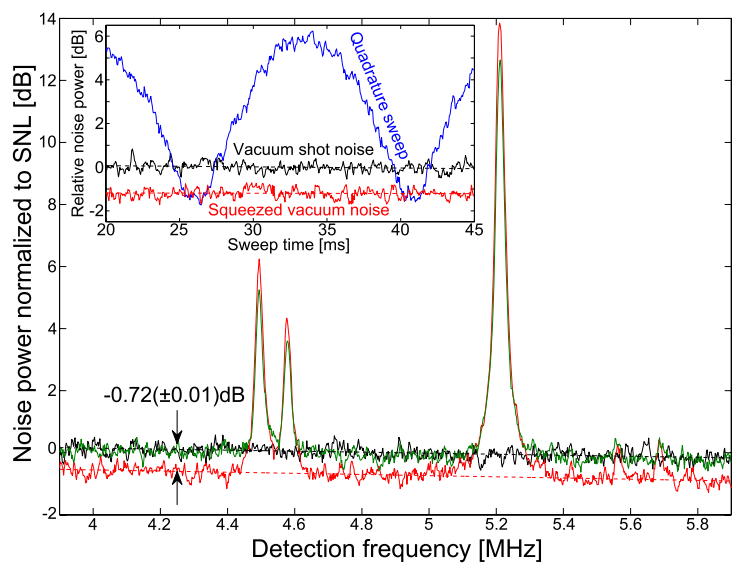

Fig. 3. Optomechanically transduced vibration spectrum, measured without (green) and with squeezed light (red), yielding a transduction sensitivity reduced below the SNL (black). All traces are averaged over 10 samples, recorded with $\mathrm{RBW}=10 \mathrm{kHz}$ and VBW $=100 \mathrm{~Hz}$. Inset: Characterization of the transmitted vacuum squeezed state. All traces were recorded in zero span mode at a detection frequency of $4.9 \mathrm{MHz}$, $\mathrm{RBW}=100 \mathrm{kHz}$, and $\mathrm{VBW}=100 \mathrm{~Hz}$. The detector dark noise ( $25 \mathrm{~dB}$ below shot noise) has not been subtracted. 
tuning of the optical cavity resonances and a fine frequency tuning of the main laser, is used to bring the probe light on resonance with an optical toroid mode with $\kappa / 2 \pi=180 \mathrm{MHz}$ (FWHM). Based on a power transmission measurement the estimated coupling parameter [14] is $\eta_{c}=0.025$, corresponding to operation in the highly undercoupled regime. Figure 3 shows the recorded spectral noise power of the balanced homodyne signal with the LO phase locked for phase quadrature detection, in the case of coherent and phase squeezed probe states. Transduced signals from three mechanical vibration modes at frequencies in the range $4-5.8 \mathrm{MHz}$ are visible in the spectrum, and most importantly the background noise floor is reduced by $-0.72( \pm 0.01) \mathrm{dB}$ below SNL when squeezed light is injected. The reduction was evaluated by linear fits to the recorded noise levels (dashed lines). All traces were recorded with probe carrier and LO powers of $20 \mu \mathrm{W}$ and $1.2 \mathrm{~mW}$, respectively. The variation in peak levels for the two probing schemes is attributed to different taper-toroid coupling strengths. Noise squeezing in the transduction signal was observed above $3.5 \mathrm{MHz}$ extending the full $20 \mathrm{MHz}$ bandwidth of the OPA.

In conclusion, we have experimentally demonstrated a quantum enhancement of the optomechanical transduction sensitivity using squeezed states of light to probe the mechanical vibrations of a tapered fibercoupled microtoroidal resonator. A transduction sensitivity $-0.72 \mathrm{~dB}$ below SNL was achieved, primarily limited by losses in the tapered fiber. We are confident that a $3 \mathrm{~dB}$ improvement of the mechanical transduction sensitivity is attainable through further optimization of the tapered fiber production, shielding and nitrogenpurging of the taper-toroid region, and increased homodyne detection efficiency by use of high-quantum efficiency diodes. Reaching this level of impact would render our scheme feasible for practical implementations. One immediate application would be to improve the efficiency of optoelectromechanical feedback cooling, where the cooling limit is set by the SNR of the transduction signal [19]. It is important to note that the demonstrated quantum-enhancement technique not only applies to cavity optomechanics in the undercoupled regime. In general, the best transduction sensitivity is achieved at critical coupling, but since $\Omega_{m} / \kappa \ll 1$ in the discussed system, working in the undercoupled regime is favorable, in order to minimize degradation of squeezing at relevant frequencies. However, a sideband-resolved system with $\Omega_{m} / \kappa>1$ [ $\underline{15}$ ] can in principle be operated in the critical-coupling regime with negligible loss of squeezing, yielding an optimal sensitivity enhancement.

This research was funded by the Danish Council for Independent Research (Sapere Aude program) and the Lundbeck Foundation. W. P. Bowen acknowledges funding by the Australian Research Council Centre of Excellence CE110001013 and Discovery Project DP0987146. Device fabrication was undertaken within the Queensland Node of the Australian Nanofabrication Facility.

\section{References}

1. D. Rugar, R. Budakian, H. J. Mamin, and B. W. Chui, Nature 430, 329 (2004).

2. S. Forstner, S. Prams, J. Knittel, E. D. van Ooijen, J. D. Swaim, G. I. Harris, A. Szorkovsky, W. P. Bowen, and H. Rubinsztein-Dunlop, Phys. Rev. Lett. 108, 120801 (2012).

3. J. D. Teufel, T. Donner, D. Li, J. W. Harlow, M. S. Allman, K. Cicak, A. J. Sirois, J. D. Whittaker, K. W. Lehnert, and R. W. Simmonds, Nature 475, 359 (2011).

4. A. H. Safavi-Naeini, J. Chan, J. T. Hill, T. P. M. Alegre, A. Krause, and O. Painter, Phys. Rev. Lett. 108, 033602 (2012).

5. C. M. Caves, Phys. Rev. Lett. 45, 75 (1980).

6. H. Rokhsari, T. J. Kippenberg, T. Carmon, and K. Vahala, Opt. Express 13, 5293 (2005).

7. G. I. Harris, U. L. Andersen, J. Knittel, and W. P. Bowen, Phys. Rev. A 85, 061802 (2012).

8. C. M. Caves, Phys. Rev. D 23, 1693 (1981).

9. M. Xiao, L.-A. Wu, and H. J. Kimble, Phys. Rev. Lett. 59, 278 (1987).

10. T. Eberle, S. Steinlechner, J. Bauchrowitz, V. Händchen, H. Vahlbruch, M. Mehmet, H. Müller-Ebhardt, and R. Schnabel, Phys. Rev. Lett. 104, 251102 (2010).

11. The LIGO Scientific Collaboration, Nat. Phys. 7, 962 (2011).

12. M. A. Taylor, J. Janousek, V. Daria, J. Knittel, B. Hage, H.-A. Bachor, and W. P. Bowen, Nat. Photonics 7, 229 (2013).

13. A. F. Pace, M. J. Collett, and D. F. Walls, Phys. Rev. A 47, 3173 (1993).

14. A. Schliesser and T. J. Kippenberg, Advances In Atomic, Molecular, and Optical Physics (Elsevier, 2010), Vol. 58, pp. 207-323.

15. A. Schliesser, O. Arcizet, R. Reviére, G. Anetsberger, and T. J. Kippenberg, Nat. Phys. 5, 509 (2009).

16. O. Glöckl, "Quantenverschränkung und interferometrie für die quanteninformationsverarbeitung," Ph.D. thesis (Friedrich-Alexander-Universität, 2005).

17. M. Lassen, M. Sabuncu, A. Huck, J. Niset, G. Leuchs, N. J. Cerf, and U. L. Andersen, Nat. Photonics 4, 700 (2010).

18. M. Fujiwara, K. Toubaru, and S. Takeuchi, Opt. Express 19, 8596 (2011).

19. K. H. Lee, T. G. McRae, G. I. Harris, J. Knittel, and W. P. Bowen, Phys. Rev. Lett. 104, 123604 (2010). 